\title{
José-Esteban Pérez García*
}

\section{LA INDUSTRIA DE LA CONSTRUCCIÓN NAVAL, LA UNION EUROPEA Y ELSIGLO XXI}

Los mares y océanos ocupan aproximadamente el 71 por 100 de la superficie del planeta y por ellos circula entre el 80 y el 90 por 100 del volumen del comercio mundial. La comprensión de la importancia estratégica de su utilización en un mundo globalizado, tanto en lo que se refiere al transporte de bienes de todo tipo como a la explotación de sus recursos, es vital para la humanidad y su desarrollo. Este artículo se refiere a la industria que fabrica los elementos que hacen posible su función y trata de explicar, tanto la naturaleza del sector como el devenir del comportamiento de las naciones con relación a sus estrategias, haciendo hincapié en el que atañe a la Unión Europea y a su cada vez más deteriorada posición internacional, fundamentalmente en relación con esta específica capacidad industrial tan ligada, estratégicamente, no solo al comercio, sino también a la aptitud soberana para asegurar los suministros imprescindibles en caso de una improbable pero no imposible necesidad.

Palabras clave: comercio marítimo, construcción naval, mercado cíclico, política industrial, transporte, recursos marinos, estrategia, competitividad.

Clasificación JEL: L22, L92.

\section{Introducción}

El perfil geográfico de la Tierra y la naturaleza de los intercambios entre sus habitantes convirtieron desde la antigüedad a la navegación en un sistema indispensable y adecuado para enviar personas y mercancías de un lugar a otro; especialmente porque la mayoría de las concentraciones humanas se han localizado

\footnotetext{
* Presidente del Comité de Asuntos Marítimos y Marinos del Instituto de la Ingeniería de España, Ex Vicepresidente Europeo del Grupo de Construcción Naval de la OCDE (WP6). Académico de número de la Real Academia de la Mar. Ingeniero Naval. Ex Director Gral. de la Asociación de Constructores Navales Europeos (AWES-CESA, hoy Sea Europe).
}

con preferencia en las orillas de los océanos, mares, lagos y ríos importantes.

Este escenario ha visto cómo crecía el volumen de sus actividades a lo largo de los tiempos y aumentaban las transacciones comerciales y de otros tipos por vía marítima de manera proporcional al ritmo del desarrollo económico mundial, impulsado a su vez por la globalización de la economía.

El vehículo que posibilita estas transacciones y sus actividades económicas conexas, y no solo ellas, es el buque, en su acepción más amplia.

Nos referimos aquí al transporte marítimo de mercancías de todo tipo, a la exploración y explotación de los 
recursos marinos, tanto vivos como fósiles, a las necesidades de defensa marítima y costera de las naciones, y a múltiples actividades científicas y de investigación.

Por tanto, el buque ha sido y es un elemento esencial en el desarrollo de la economía global, y su construcción y tecnología están ampliamente involucradas por los entornos económicos, financieros, operativos, políticos y medioambientales, dando lugar a una industria de carácter estratégico. Así lo han entendido muchos países a lo largo de los tiempos, aunque hay que reconocer que unos mejor que otros.

\section{Características fundamentales de la industria de construcción naval}

Una de las características más destacables de la industria de la construcción naval, y específicamente de la que sirve al transporte de mercancías y a la explotación de recursos marinos, es la naturaleza global de su mercado, con alguna excepción que será mencionada más adelante. En este escenario global se produce una elevada competencia internacional que deriva de la explotación del producto, el buque, en un mercado de elevada concurrencia si se le compara con otros medios de transporte y con las industrias que los fabrican.

A diferencia de otros medios de transporte como la aviación, el ferrocarril o el transporte por carretera, que generalmente gozan de una fabricación seriada, en la industria de la construcción naval tal cosa no se produce, y si lo hace, las series son extraordinariamente cortas. De hecho, es probablemente la única industria de fabricación de medios de transporte en la que el prototipo se vende (no se construye un modelo contra almacén para luego ponerlo en el mercado), lo que ocasiona una enorme dificultad para concurrir a los beneficios que los países otorgan a las inversiones en investigación, desarrollo e innovación 1 .

\footnotetext{
1 FRASCATI MANUAL (2015). Guidelines for Collecting and Reporting Data on Research and Experimental Development.
}

Tradicionalmente la industria de la construcción naval se ha distinguido por una producción de buques singulares de muy alto valor unitario, en la que lo que podríamos llamar «prototipo» es el propio buque objeto de un contrato de compraventa entre el armador y la empresa constructora (el astillero). La duración del periíodo de construcción, intrínsecamente larga dado el volumen del producto, el valor de la inversión y el hecho ya mencionado de que no se suele construir en serie, muy dependiente también de los ciclos del mercado y los desfases entre oferta y demanda típicos del negocio marítimo, puede afectar de manera importante a los acaecimientos y provocar modificaciones en el coste de la construcción. Coste que es clave para el astillero, al igual que la frecuente volatilidad de los ingresos provocada por las oscilaciones en el mercado de fletes, y que son a su vez claves para el armador que encarga los buques.

Todo ello configura un tipo de industria con una producción de naturaleza cíclica y muy influida, debido a su carácter estratégico, por las políticas industriales sectoriales de los grandes países o áreas constructoras que obviamente pueden provocar y provocan alteraciones en el escenario de la competencia internacional.

La industria de la construcción naval ha evolucionado sensiblemente desde su antigua consideración de «mano de obra intensiva» hacia "capital y tecnología intensivos», y a lo largo de esta evolución se ha ido transformando en una industria de síntesis en la que la empresa que entrega el buque al armador aporta entre el 30 y el 20 por 100 del valor del producto, aunque su responsabilidad finalista responda de la totalidad. El valor añadido externo depende del tipo de buque del que se trate. En el caso de los buques mercantes podemos colocar en los extremos y como ejemplo de menor a mayor valor añadido exterior a los buques de transporte de graneles sólidos en el extremo inferior (mineraleros, graneleros, etc.) y a los buques de pasaje de cruceros en el extremo superior.

Como orientación, los precios actuales de los mayores buques de estos dos tipos se mueven 
respectivamente en torno a los 50.000 .000 de dólares (USD) y los 1.300 millones USD².

Los astilleros se constituyen en fábricas con importantes departamentos de diseño y de ingeniería concurrente con sus suministradores que incorporan a la estructura del buque equipos y componentes de características diversas y de tecnologías diferenciadas. A título de ejemplo citaremos de manera indicativa como sectores integrantes del eslabonamiento en el funcionamiento y en los suministros a los astilleros: la industria siderúrgica, la industria eléctrica y electrónica, la industria metalúrgica no férrea, la de productos metálicos y no metálicos elaborados y semielaborados, equipos y maquinaria no eléctrica, hardware y software informáticos, industria química, industria de la madera y del mueble, industria textil y de los plásticos, restauración y hostelería. Igualmente el sector financiero, los servicios jurídicos y de seguros, y otros muchos que sería prolijo citar aquí.

Cuanto más complejo y de mayor valor es el buque, más crítico ha de ser el trabajo del astillero constructor en las ingenierías del proceso y del producto, ya que tiene la responsabilidad finalista de la entrega en el precio y el plazo pactados en el contrato.

Se puede apreciar, por tanto, que la industria de la construcción naval se relaciona técnica y económicamente por la vía de los suministros con un abanico de importantes y amplios sectores industriales y de servicios. Es por esto por lo que en las políticas industriales de muchos países se ha utilizado como elemento tractor del desarrollo industrial y de servicios. Esto ha sido más cierto cuanto más destacada era su naturaleza de sector de mano de obra intensiva, y especialmente en países en los que la creación de empleo industrial era una política básica. Este escenario ha ido poco a poco mutando conforme se desarrollaban productos más complejos y que requerían inversiones más cuantiosas para poder competir. Así, el factor de la mano de obra se ha reducido proporcionalmente, ocupando

2 Revista Ingeniería Naval (2017). «Coyuntura». № 963, noviembre 2017. un nivel más acorde con los tiempos, especialmente en los países del llamado "primer mundo», algunos de los cuales son los líderes globales en este sector industrial. El caso de China, como primera economía emergente en el mundo, es especial pues compatibiliza actualmente las características antigua y nueva.

La cada día más elevada utilización de tecnologías de última generación, tanto en el proyecto como en la fabricación y en las prestaciones, son elementos cruciales de competitividad. También lo son los cumplimientos de exigentes regulaciones relativas al medio ambiente, respecto de los cuales y en multitud de casos la propia industria, de la mano de su cliente, la industria naviera, camina avant la lettre dentro de las limitaciones que marca la naturaleza de los buques, tales como sus largos períodos de navegación, su necesidad de energía concentrada combinada con la importante demanda de potencia en la propulsión, el tamaño creciente de los buques, etc. Pese a todo esto, el transporte marítimo continúa siendo, con diferencia, el sistema de transporte que produce menos emisiones de gases nocivos a la atmósfera por tonelada transportada, lo que no es óbice para que, dado su enorme volumen, se esté trabajando a todos los niveles para reducir las emisiones de sustancias contaminantes y gases de efecto invernadero (GEI), $\mathrm{NO}_{x}$ y $\mathrm{SO}_{x}$. La realidad hoy día, en lo que se refiere a los GEI es que la aportación del transporte marítimo no representa más del 3 por 100 del total de origen antropogénico.

La vieja idea de que este tipo de industria era más propia de países en vías de desarrollo que se iba abandonando conforme el sector servicios aumentaba su posicionamiento en la estructura del producto interior bruto parece estar demostrando su inexactitud, sobre todo si se toma totalmente al pie de la letra.

Como se ha dicho, esta industria tiene una influencia muy significativa en el empleo y en el desarrollo industrial, lo que ha motivado, en muchos casos y en el devenir reciente de la historia, intervenciones políticas de los Estados para protegerla, provocando importantes distorsiones en el comportamiento del mercado, 
que interactuaban, además, con las propias del carácter cíclico del mismo amplificando los desequilibrios.

La existencia y la supervivencia de una industria como la que nos ocupa demanda unos niveles de inversión económica importantes, especialmente en inmovilizado, y un elevado nivel de experiencia técnica que se ponen al servicio de otra industria, la naviera, cuyo desenvolvimiento depende de la demanda de transporte, que a su vez se comporta reflejando los movimientos de los crecimientos económicos y los flujos comerciales tanto globales como regionales. También es importante resaltar que la industria naviera suele caracterizarse por un fuerte endeudamiento dado el alto coste que representa la inversión en un buque, más aún si el buque es de nueva construcción. Es habitual que la contratación de un buque de nueva construcción lleve consigo una financiación del 80 por 100 del valor del buque, por lo que las amortizaciones de la deuda influyen de manera importante en el cashflow naviero.

Generalmente, la naturaleza propia del negocio naviero provoca que el diseño de la estrategia financiera sea crucial para el proyecto completo, ya que el escenario no suele seguir los cánones con los que se trabaja en las financiaciones industriales más comunes, tales como ingresos estimables o predecibles para un período de tiempo determinado, estructuras claramente definidas, etc.

Otra de las peculiaridades de la industria de la construcción naval tiene que ver con cómo las empresas constructoras afrontan las predicciones de evolución del mercado para plantear sus estrategias, tanto técnicas como comerciales.

Dado que el transporte marítimo y su evolución reflejan bastante fielmente la del crecimiento mundial de la economía, los astilleros y sus organizaciones tratan de estudiar las predicciones que las instituciones mundiales concernidas por la evolución de la economía y el comercio tratan de diseccionar, así como los posibles flujos, tanto de materias primas como de productos elaborados o semielaborados, que constituyen el grueso de la utilización del transporte marítimo. De esta manera, y teniendo en cuenta los segmentos y las edades de la flota existente, los tamaños de los buques, su vida útil por tipos y por lo tanto la evolución esperable de los desguaces, puede intentarse hacer una proyección de los requerimientos de flota para los años subsiguientes. Esta proyección no tiene necesariamente que reflejar la demanda real, ya que hay factores que influirán en que estas predicciones se acerquen a la realidad o acaben forjando un escenario distinto al calculado.

Crisis bruscas en los crecimientos económicos, variaciones de rutas habituales producidas por situaciones externas a la navegación, crecimientos distintos de los esperados en las capacidades portuarias y sus hinterland, movimientos políticos que afectan al comercio mundial y por lo tanto a su principal vehículo, y otros muchos, hacen que la demanda real pueda diferir de los requerimientos teóricos de flota nueva estimados técnicamente por los constructores y sus organizaciones.

Resulta interesante comentar aquí algunos de los casos más llamativos de estos vaivenes que además se superponen a los ciclos habituales aumentando la importancia y las consecuencias de estos. En la primavera del año 2008, la cartera mundial de pedidos de la flota de buques portacontenedores representaba el 57 por 100 de la capacidad de la flota activa contada en número de contenedores (TEU, Twentyfoot Equivalent Unit); en la actualidad no llega al 13 por 100. En el caso de otros tipos de buques, como los de transporte de graneles líquidos (petróleo crudo, derivados, productos químicos), estos porcentajes eran y son del 42 por 100 y del 12 por 100 respectivamente; en el caso de los graneles sólidos, del 60 por 100 y del 7,6 por 100, contados en toneladas de peso muerto (TPM); y el de los buques de transporte de gas natural licuado, del 58 por 100 y 28 por 100 , contados en metros cúbicos (MC). La inversión total en nuevos contratos que había ascendido a 263.100 millones USD durante el año 2007, que ya empezó a caer en 
2008, con 166.000 millones USD, cayó a 30.000 millones en 2009 (el peor año para el crecimiento económico mundial) y a final de agosto de 2017 se encuentra en 40.000 millones USD.

Un abultamiento de las carteras de pedidos como el mencionado desplaza hacia el futuro las entregas de importante número de buques, y algunos astilleros empiezan a aumentar su capacidad mientras aparecen otros de nuevo cuño que tratan de entrar en el mercado de construcción de aquellos tipos de buques para los que las barreras de entrada, tanto de carácter técnico como constructivo puedan ser bajas, produciendo así un desequilibrio mayor en el mercado. En muchos de estos casos se ha dado además la circunstancia de que la viabilidad financiera de las empresas era muy precaria, "colgada» de una continuidad en la obtención de nuevos contratos (comportamientos piramidales) que no suele darse, debido al carácter cíclico del negocio. Los retrasos en las fases de la ejecución de las obras motivados por la falta de experiencia llevaban consigo importantes desfases en los pagos del armador, rompiendo así el esquema «piramidal» en el que se basaba el constructor. En muchas ocasiones, además, parte de los armadores que habían contratado padecían de un vicio estructural semejante, aumentando así la fragilidad de las operaciones.

Un ejemplo reciente de estas situaciones se ha dado en China donde, al calor de la demanda creciente que hemos mencionado anteriormente, nacieron astilleros relativamente pequeños y precariamente dotados, de propiedad privada o semiprivada. Cuando se precipitó la crisis y los pedidos dejaron de llegar, empezaron a sufrir los problemas expuestos antes, no cumpliendo los plazos, imposibilitados de financiar su circulante y pagar a los suministradores y soportando cancelaciones e impagos por parte de los clientes. Conforme fue avanzando la crisis, estos astilleros faltos de consolidación fueron cerrando. La industria de la construcción naval china redujo su desproporcionada capacidad, manteniendo casi intacta la de las grandes compañías constructoras de propiedad estatal, que en casos como el presente suelen ser alimentadas con pedidos cuantiosos en número y tamaño, producidos por compañías estatales con independencia de la situación global del equilibrio oferta-demanda de transporte marítimo. Este comportamiento no ha sido exclusivo de China ni en el presente ni en el pasado, sobre todo teniendo en cuenta el carácter estratégico que muchos países han otorgado y otorgan a la industria de la construcción naval, además de considerarla en fases de desarrollo industrial, como una actividad de carácter económico "polar» y por lo tanto locomotora de la industrialización de las zonas donde se implantaba o existía.

La razón estratégica, en la que incidiremos más adelante, tiene que ver con la intención de mantener una soberanía tecnológica sobre un factor vital para el país de que se trate: la seguridad de obtener sus aprovisionamientos vitales y estratégicos dependientes del transporte para los que la vía marítima es clave. No se trata tanto de poseer una importante flota controlada por armadores propios, que también, como de mantener la tecnología y la capacidad de construir esos medios de transporte que aseguren los aprovisionamientos en épocas «tormentosas» que la historia ha enseñado que, aunque inesperadas, no dejan de existir. Obviamente, mantener una industria de construcción y una flota capaces de competir con éxito en situaciones normales es la mejor manera de asegurarse lo anterior; más aún cuando las condiciones y los comportamientos distan mucho de lo que se ha dado en llamar level playing field, comportamiento que en el ámbito de la competencia internacional no ha existido nunca, como veremos más adelante. De hecho, la historia de la industria de la construcción naval es un devenir de proteccionismos, ayudas y subsidios. Las condiciones normales de competencia propias de un mercado abierto no se han dado nunca. $Y$ esto, unos lo han entendido mejor que otros, dependiendo en muchos casos de la longitud de miras de sus políticas industriales. 
3. Evolución y reparto de la industria de construcción naval mundial

La industria de referencia evoluciona al ritmo que marca el comercio mundial, que a su vez refleja el del crecimiento económico mundial, y lo hace de la manera irregular que históricamente han marcado sus ciclos pero, de una manera u otra, los constructores han ido respondiendo, a veces con ciertos desfases, a las necesidades reales de buques, a su vez imprescindibles para dar respuesta a los requerimientos de los flujos comerciales.

Podemos aquí glosar la frase: Navegare necesse est, vivere non necesse, atribuida a Pompeyo como arenga a sus marineros, que asustados por el amenazador estado de la mar, eran renuentes a subir a bordo de su barco, que transportaba trigo hacia Roma.

En el Cuadro 1 se presenta, en porcentaje, el reparto de entregas de buques en el mundo, medida en toneladas de registro bruto GT, que enseña cómo en casi un siglo el centro de gravedad de la industria ha ido paulatinamente abandonando Europa hacia la zona Asia-Pacífico.

Durante los primeros años del período representado, era la construcción naval europea, mayoritariamente localizada en Reino Unido, la que dominaba el mercado. Esta situación se mantuvo sin grandes cambios, con un ligero aumento de Japón a costa de Europa, hasta la Segunda Guerra Mundial, en la que se desató la construcción naval de EE UU (estamos tratando solo la construcción de buques de transporte, no de buques de guerra), que llegó a representar durante el tiempo que duraron las hostilidades el 80 por 100 de las entregas mundiales en tonelaje. Estos buques eran necesarios para asegurar los suministros de todo tipo en el Pacífico y en el Atlántico dada la extensión geográfica del conflicto. Nótese también que la transición del tipo de transporte marítimo hacia unidades de gran tonelaje sucedería muchos años después.

Muchos de aquellos buques, realmente no construidos para la durabilidad en tiempo de paz, fueron desguazados al fin de la guerra, y otros comprados por armadores privados que los explotaron durante algún tiempo.

En la década de los años cincuenta del pasado siglo, la industria de la construcción naval europea empezó a recuperar cuota de mercado mientras se asistía al despegue de la capacidad de construcción japonesa, sobrepasando a la europea que empezó a languidecer en los ochenta; época en la que comenzaba el despegue de la industria de Corea del Sur, que llegó a liderar el mercado mundial justamente al empezar el Siglo XXI. La construcción naval europea siguió cayendo hasta convertirse en la actualidad en una presencia casi testimonial en casi todos los segmentos del mercado.

Al principio de la segunda década de este siglo irrumpe agresivamente la industria china, propulsada por políticas industriales extraordinariamente dirigidas para colocarse hoy en cabeza en volumen de producción aunque no todavía en valor.

Tras este repaso al devenir histórico de la industria que nos ocupa, conviene hacer algunas consideraciones. Con excepción de la discontinuidad correspondiente a la Segunda Guerra Mundial, puede observarse que las transiciones entre las diferentes cuotas de mercado de los grandes constructores son relativamente suaves, con la excepción de la irrupción de China.

Desde la mencionada guerra, la demanda de construcción de buques a muy largo plazo se mantiene positiva como corresponde a un mundo que va globalizando su economía y en el que, con pequeñas oscilaciones, el crecimiento económico mundial mantiene un continuado ascenso, aunque en determinadas circunstancias algunas zonas se hayan visto afectadas por crisis económicas agudas (la permanente pendiente positiva del crecimiento económico mundial solo acusó un pequeño retroceso en el año 2009). Sin embargo, y como se ha indicado en el apartado anterior, la demanda a corto plazo, y por diversas razones, puede presentar alteraciones importantes, algunas debidas a factores externos, como fue el caso de la crisis del petróleo de 1973 o la crisis financiera que comenzó en 2008, y que por su carácter externo son 


\begin{tabular}{|c|c|c|c|c|c|c|}
\hline \multicolumn{7}{|c|}{$\begin{array}{c}\text { ENTREGAS DE BUQUES NUEVOS POR PAÍSES / ÁREAS EN \% ANUAL } \\
\text { (Medidas en GT) }\end{array}$} \\
\hline Años & Europa & Japón & Corea S. & R. P. China & EE UU & Otros \\
\hline $1925 \ldots$ & 90 & 3 & 0 & 0 & 5 & 2 \\
\hline $1930 \ldots \ldots \ldots \ldots \ldots \ldots$ & 83 & 5 & 0 & 0 & 10 & 2 \\
\hline $1935 \ldots$ & 86 & 11 & 0 & 0 & 2 & 1 \\
\hline 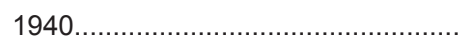 & 82 & 8 & 0 & 0 & 6 & 4 \\
\hline 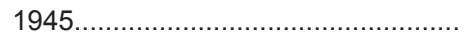 & 15 & 0 & 0 & 0 & 80 & 5 \\
\hline 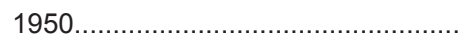 & 75 & 11 & 0 & 0 & 12 & 2 \\
\hline 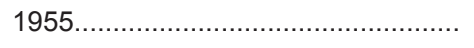 & 85 & 12 & 0 & 0 & 1 & 2 \\
\hline $1960 \ldots \ldots \ldots \ldots \ldots \ldots$ & 67 & 20 & 0 & 0 & 5 & 8 \\
\hline 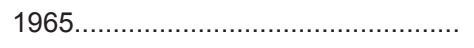 & 55 & 42 & 0 & 0 & 2 & 1 \\
\hline 1970 & 45 & 47 & 0 & 0 & 3 & 5 \\
\hline 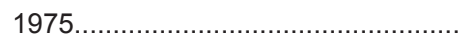 & 42 & 48 & 2 & 0,5 & 2 & 5,5 \\
\hline $1980 \ldots \ldots \ldots \ldots \ldots \ldots \ldots$ & 23 & 47 & 4 & 0,5 & 4,5 & 21 \\
\hline $1985 \ldots \ldots \ldots \ldots$ & 17 & 52 & 17 & 2 & 0 & 12 \\
\hline 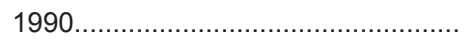 & 20 & 40 & 22 & 3 & 0 & 15 \\
\hline 1995 & 18 & 40 & 27 & 5 & 0 & 10 \\
\hline 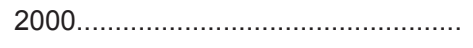 & 15 & 32 & 36 & 7 & 2 & 8 \\
\hline 2005 & 12 & 26 & 43 & 8 & 2 & 9 \\
\hline $2010 \ldots \ldots \ldots \ldots \ldots \ldots$ & 5 & 21 & 35 & 38 & 0,2 & 0,8 \\
\hline 2015 & 2 & 19 & 36 & 37 & 0,5 & 5,5 \\
\hline 2016 & 4 & 20 & 38 & 33,3 & 0,45 & 4,25 \\
\hline
\end{tabular}

difícilmente predecibles desde la óptica de la industria de la construcción naval. En cualquier caso, ambas actuaron indirectamente en la amplificación de los ciclos del mercado de los astilleros. La primera porque, además de otras cosas, afectaba directamente al transporte de petróleo crudo, y por reflejo al de otros productos energéticos, como los derivados del petróleo, productos químicos líquidos y al carbón, y obviamente al precio de los combustibles para propulsión, uno de los costes de explotación más importantes en el transporte marítimo. De hecho, la situación provocó un aumento de demanda de buques mineraleros, así como una corriente de cambio de los sistemas de propulsión, desde motores de combustión interna a turbinas de vapor con calderas de carbón de lecho fluido.
En un mercado cíclico, como es el de la construcción naval mercante o la dedicada a la explotación de los recursos marinos, las crisis hacen desaparecer a los contendientes menos preparados, dejando espacios para los que han sobrevivido mejor y se han preparado de manera más idónea para la siguiente fase. La pura contracción de la oferta por simple reducción de capacidad de construcción, tanto en fuerza laboral como en instalaciones, no ha probado ser una receta aceptable para permanecer en el mercado, especialmente si en las fases de crecimiento esos astilleros no han profundizado en avances tecnológicos, en mejoras continuas en la ingeniería de procesos y en la productividad, ni en estrategias comerciales más próximas al mundo naviero. 
El cambio del centro de gravedad de la industria de la construcción naval en el mundo no solo ha estado motivado por el desarrollo industrial y los intereses marítimos de algunas naciones de la zona Asia-Pacífico, sino por una política falta de acierto en Europa, y especialmente en la Unión Europea.

Para proporcionar al lector una visión más gráfica del escenario actual, en el Cuadro 2 se presentan las cifras que indican los valores económicos de las carteras de pedidos, de las contrataciones y de las entregas de las áreas y países más importantes en esta industria, al final del año 2016.

Como se puede apreciar, el valor de la cartera de pedidos de los países asiáticos representa un 72 por 100 del total mundial. La cartera de pedidos asiática está mayoritariamente compuesta por la de tres países: China, Corea del Sur y Japón. El conjunto de las cifras del cuadro requiere una explicación, especialmente en lo que se refiere a la construcción naval europea (no solo la UE) y la aparición en el cuadro de Italia y Alemania. Ambos países suman un apreciable volumen de contratación en el año 2016, tendencia que se continúa en 2017, constituida de forma muy mayoritaria por el segmento de buques de pasaje de crucero, segmento en el que los astilleros públicos italianos Fincantieri (que ahora controlan también el mayor astillero francés), y los privados alemanes Meyerwerft, propietarios también del mayor astillero dedicado a este tipo de buques en Finlandia, se reparten prácticamente el mercado de construcción de buques de crucero del mundo.

Como se puede ver, existen importantes diferencias en las comparaciones entre las contrataciones y las entregas. Las carteras de pedidos de los astilleros de cruceros se tardan en consumir bastante más por unidad que las del resto de los tipos de buques y de ahí las diferencias entre el valor entregado por los países asiáticos y los europeos.

Se da la circunstancia de que el segmento de buques de pasaje de crucero es el que menos ha sufrido en el período de crisis en lo que se refiere al ritmo de encargo de construcción de nuevos buques y de hecho

\section{CUADRO 2}

VALOR DE LA CARTERA, CONTRATOS, ENTREGAS, 2016

(En millones de dólares)

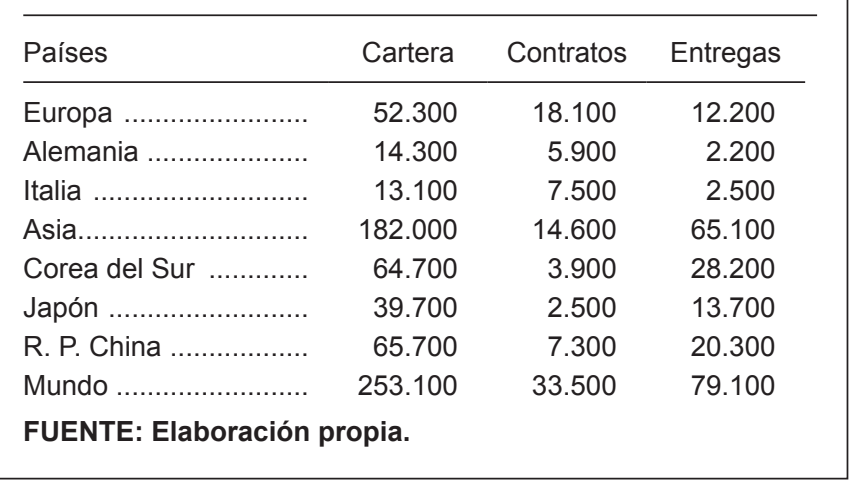

se puede decir que no ha sufrido, mientras todos los demás, y muy especialmente el sector offshore, sí lo han hecho.

Esta situación de práctico duopolio en el mercado de la construcción de cruceros se rompe en 2017 con la contratación por parte de los grandes astilleros públicos chinos de sus primeros grandes buques de cruceros para uno de los principales grupos navieros internacionales. Todo ello con el apoyo tecnológico del grupo italiano Fincantieri. La irrupción de la industria china en uno de los segmentos con barreras de entrada más altas se acomete gracias a la política industrial dirigida del Gobierno chino, política que ha sido una constante desde el principio del despegue económico del país.

En el Cuadro 3 se presentan algunos de los indicadores más representativos de la evolución de la industria mundial de construcción naval comparados con la del transporte marítimo desde 2010, aunque a modo de contraste se muestran también los valores de esos indicadores en el año 2007, pico del ciclo del que venimos hablando.

Por contraste con la caída espectacular de las cifras que marcan la evolución negativa de la industria, el volumen del transporte marítimo se mantiene en un crecimiento moderado durante todo el tiempo, aunque con 


\section{CUADRO 3}

INDICADORES DE LA CONSTRUCCIÓN NAVAL MUNDIAL Y TRANSPORTE MARÍTIMO

\begin{tabular}{|c|c|c|c|c|c|c|c|c|}
\hline Indicadores & 2007 & 2010 & 2011 & 2012 & 2013 & 2014 & 2015 & 2016 \\
\hline Cartera de pedidos. Millones CGT ........................ & 190,0 & 139,8 & 115 & 92,8 & 103,9 & 115,1 & 109,3 & 86,2 \\
\hline 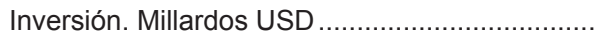 & 263,1 & 71,7 & 90,4 & 80,8 & 103,8 & 101,2 & 69,0 & 33,5 \\
\hline Contratación. Millones CGT ................................ & 85,5 & 33,8 & 28,1 & 21,3 & 48,7 & 39,7 & 33,6 & 11,2 \\
\hline Entregas. Millones de CGT ............................... & 34,0 & 48,0 & 48,0 & 45,3 & 36,6 & 34,7 & 36,7 & 34,5 \\
\hline Transporte marítimo. Millones Tm... & 7.360 & 8.255 & 8.784 & 9.838 & 10.176 & 10.529 & 10.790 & 11.536 \\
\hline
\end{tabular}

NOTA: *CGT = Toneladas brutas compensadas. Las toneladas brutas compensadas corrigen las habituales unidades que se utilizan técnicamente en el diseño y la construcción de buques con coeficientes dependientes del valor de los buques por tipos y de la tecnología y complejidad incorporadas.

FUENTE: CLARKSON RESEARCH (2017).

ciertos altibajos. Según los datos de la Conferencia de las Naciones Unidas sobre el Comercio y el Desarrollo (UNCTAD), el ritmo de crecimiento del volumen de mercancías transportadas por vía marítima descendió desde el 4,4 por 100 en 2012 hasta el 2,6 por 100 en 2016, mientras que en el año 2017 todo apunta a una mayor velocidad de crecimiento del comercio marítimo, previsión que se cifra en un 4,3 por 100 medida en toneladas-milla. Todo ello es congruente con el crecimiento del PIB de la economía mundial, que fue de 3 por 100 en 2016, con una previsión del 3,5 por 100 en 2017 y del 3,7 por 100 en $2018^{3}$.

Los datos que aparecen en el Cuadro 3 dan una idea clara del comportamiento completamente cíclico exacerbado de la industria de la construcción naval, en la que se amplifican las magnitudes de los ciclos por la combinación de los comportamientos de las dos partes en litigio: el negocio naviero y los fabricantes de buques, en contraste con la evolución más pausada y más uniforme de la demanda real de transporte marítimo.

Finalizando 2017, todo parece apuntar a una mejoría de la situación tras el duro ajuste realizado en estos últimos años; sin embargo, no todos los segmentos

\footnotetext{
3 OCDE (2017). Interim Economic Outlook. Septiembre.
}

del negocio marítimo suelen evolucionar con el mismo grado de progresión, cosa que por otra parte ha sucedido siempre.

Es conveniente no tratar al conjunto de la industria de construcción naval como un todo homogéneo cuando nos ocupamos de sus aspectos económicos, incluso cuando se trata de la parte que tiene que ver con el transporte de mercancías y la explotación de recursos marinos, que son el objeto principal de este artículo. El transporte marítimo tampoco lo es, y son las alteraciones de los diferentes tipos de tráficos los que marcan las diferencias entre los ciclos de la industria que componen el ciclo general, y que a menudo compensan esas diferencias entre sí contribuyendo a un crecimiento mantenido, como ya se ha visto. Así, la construcción de buques que transportan productos energéticos (petróleo crudo, derivados, gases licuados, carbón, etc.) responde a la situación de las necesidades de energía y de los precios de la misma, cosa que también sucederá con los buques y artefactos cuyo empleo está directamente relacionado con la explotación de los fondos marinos, por un lado, y de los que se ocupan de la producción de energía limpia, tales como la eólica marina, olas, corrientes, mareas, etc., que también dependen del crecimiento 
de la producción de energías no contaminantes. En la fabricación de buques para el transporte de graneles sólidos, como el carbón ya citado, los minerales, especialmente el hierro, su demanda obedecerá al crecimiento industrial en la producción de bienes férreos (automóviles, elementos ferroviarios, material de defensa, etc.), y el grano, su tráfico y volumen del mismo depende de la bondad de las cosechas en lugares distantes. En los productos elaborados o semielaborados, mayoritariamente transportados en buques portacontenedores o en buques para cargas rodadas, su incidencia en el mercado de la construcción naval es muy alta, ya que la carrera para disponer de buques cada vez más grandes está cambiando la capacidad física de los astilleros que se mueven en este mercado. Resulta interesante revivir las circunstancias que motivaron en su momento el descomunal aumento de tamaño de los nuevos buques portacontenedores. Las razones más importantes fueron dos: una obvia, los beneficios del efecto de escala, y otra, el aumento del precio de los combustibles, que obligaba a reducir la velocidad para consumir menos por milla navegada. Conviene recordar que en 2007, con un precio del barril de petróleo (Brent) de 73 USD, las toneladas de fueloil y de gasoil (precio Rotterdam) costaban 347 y 571 USD respectivamente, y que ya en 2012, con 111 USD/barril de petróleo, los respectivos precios de los combustibles mencionados llegaron a 585 y 920 USD/tonelada.

En relación con lo anterior y desde el punto de vista del diseño de los nuevos buques así como de los que se entregarán por los astilleros a medio plazo, hay que reseñar que las limitaciones aceptadas internacionalmente por la Organización Marítima Internacional (OMI) sobre emisiones a la atmósfera de óxidos de nitrógeno $\left(\mathrm{NO}_{x}\right)$ y de azufre $\left(\mathrm{SO}_{x}\right)$ que inciden directamente en la contaminación y gases de efecto invernadero como el anhídrido carbónico $\left(\mathrm{CO}_{2}\right)$ y el metano $\left(\mathrm{CH}_{4}\right)$ tendrán un papel determinante en el mercado de la construcción naval, y un reflejo también significativo en el de las reparaciones y actividades de las transformaciones de buques.
Las limitaciones impuestas se derivan de regulaciones en marcha, como el Indice de Eficiencia Energética en el Diseño (EEDI por sus siglas en inglés), las Áreas de Control de Emisiones a la Atmósfera (ECA Zones), así como la Convención de tratamiento del agua de lastre.

La más exigente a corto plazo es la eliminación del azufre, que debe pasar del 3,5 por 100 en masa, a 0,50 por 100 en el año 2020, a cuyo cumplimiento se puede llegar por dos caminos: mediante la utilización de combustibles refinados tipo gasoil como ya se hace en las zonas ECA, o mediante la colocación de dispositivos (scrubbers) que eliminen el azufre en la poscombustión. Otras soluciones tales como la mezcla de destilados con fueloil parecen menos recomendables por sus posibles inestabilidades en la composición de las emisiones. Será el armador en función de sus tráficos y el valor de las inversiones combinadas con la vida útil del buque quien deberá elegir, y la industria de construcción de buques la que debe conseguir dar la mejor respuesta técnica posible, teniendo en cuenta que las prestaciones deben cumplir, no solo con el EEDI sino también con el Índice Operacional de Eficiencia Energética (EEOI por sus siglas en inglés), y el Ship Energy Efficiency Management Plan (SEEMP), que solicita del armador o del operador, en su caso, considerar nuevas prácticas o tecnologías cuando quieran mejorar el rendimiento del buque.

En estas condiciones es altamente probable, por los cambios que se producirán en la demanda de productos refinados, que debe aumentar, al igual que en la demanda de fuel pesado, que debe disminuir, que los precios del combustible a utilizar aumenten sensiblemente.

La industria de construcción naval en el mundo tiene en ese sentido un reto por delante muy importante.

A la vista del porvenir ya bastante inmediato, el gas natural se perfila como el sustituto «natural» como combustible para la propulsión de los buques, a utilizar en la forma de gas natural licuado (GNL), ya que no traslada contaminantes de nitrógeno ni de azufre; está formado mayoritariamente por metano $\left(\mathrm{CH}_{4}\right)$. Pero hay un camino largo todavía para poder 
disponer geográficamente de posibilidades de aprovisionamiento en las cabeceras de las rutas. El GNL parece, y así lo opinan las mayorías, el combustible puente entre lo que ahora se utiliza y el uso masivo de energías limpias en la navegación. En cualquier caso hay que tener en cuenta que aunque se controle y disminuya la emisión de $\mathrm{NO}_{x}$ y de $\mathrm{SO}_{x}$ por tonelada-milla de buque navegando y cumpliendo estrictamente los índices de las regulaciones antes mencionadas, lo que finalmente importa es la emisión de esos gases por el transporte marítimo a nivel global, dado que lo previsto es un aumento del crecimiento económico global y consecuentemente del tráfico marítimo. Así pues, aunque los buques cumplan de manera individual, el conjunto aumentado de las flotas puede llegar a compensar parte de la reducción conseguida.

\section{La Unión Europea y la industria de la construcción naval mercante}

Como se desprende de los datos antes expuestos, la industria de la construcción naval en el mundo no está muy repartida. Muy pocos países copan la inmensa mayoría de la producción mundial. De hecho son tres aquellos que en la actualidad suministran al mundo los elementos más importantes para que se mantenga y crezca el volumen del comercio, y los tres, China, Corea del Sur y Japón, han utilizado políticas industriales específicas para el crecimiento y/o el mantenimiento de una industria fuerte; y no siempre el comportamiento ha sido el de competir con las reglas del libre mercado. Quizá sea interesante analizar cómo los países punteros han considerado el sector industrial que nos ocupa. Lo han hecho como un sector industrial dinámico en crecimiento y no en declive aunque esté aquejado de fuertes ciclos de amplitud variable. Ellos han apostado por una permanencia duradera en el mercado, conscientes de su valor estratégico, pues no de otra manera puede considerarse la capacidad para construir una herramienta vital para el comercio así como para mantener la soberanía tecnológica que permita construir la herramienta que en momentos turbulentos o extremos permita asegurar sus propios suministros. Siguiendo este sendero, han ido consiguiendo aumentar las barreras de entrada en la industria para los competidores exteriores y muy especialmente para los europeos que creían jugar en un verdadero level playing field, cuando no era así, y más aún cuando la demanda europea era libre para contratar con cualquier constructor del mundo con ayudas de Estado europeas, mientras la demanda naviera de la competencia, creciente como su propio PIB y su actividad industrial y comercial, solo contrataba en su industria doméstica, obviamente gozando de apoyos nacionales que, aunque en algunos casos no prohibían formalmente la construcción en países externos en la práctica distaba mucho de ser así.

Otra cosa es que para conseguir ese nivel no sea conveniente restringirse a las necesidades de la demanda nacional, sino ocupar un puesto puntero en el mercado internacional, tanto en lo que se refiere al diseño y la fabricación, como a la explotación, situación clave para no caer en el autismo y en la falta de competitividad.

Obviamente, también la industria de la construcción naval de defensa es un factor importante desde el punto de vista estratégico, incluyendo las transferencias de tecnologías entre ambos sectores, que en muchos casos comparten instalaciones de fabricación.

Durante largo tiempo, los países europeos y muy significativamente los de la UE, ya desde la época de la UE de los 15, inmersos en costosos procesos de ayudas, reestructuraciones y reducciones de capacidad no siempre acompañados de los cierres exigibles en consecuencia, defendieron la idea, que empresas y Gobiernos se vendieron mutuamente como coartada, de que los astilleros europeos no podían competir en la construcción de buques en la que el contenido de la mano de obra era intensivo y el contenido tecnológico escaso. La razón esgrimida era que los competidores asiáticos, además de hacer dumping pagaban unos salarios muy bajos. Según esta filosofía, los astilleros europeos «aseguraban» una posición dominante en el segmento de buques complejos y de alto valor. 
Todo ello constituyó un gran error estratégico que generalmente no ha sido reconocido. La conquista del mercado por Japón, Corea del Sur, y más recientemente China, se ha fundamentado en fuertes políticas nacionales de fomento de las industrias que consideraban estratégicas, entre las que se contaba la construcción naval.

De esta manera se ha llegado a la situación actual, en la que ni la construcción de buques complejos de alto valor añadido, ni el volumen de sus carteras de pedidos, ni su valor son, desde hace años, patrimonio de los astilleros europeos.

Uno de los ejemplos paradigmáticos que mejor ilustra lo anterior ha sido el caso de los buques dedicados al transporte de gas natural licuado (cargamento a $160{ }^{\circ} \mathrm{C}$ bajo cero mantenido durante toda la travesía solamente con sofisticados sistemas de aislamiento). Estos buques eran considerados en el rango alto entre los de alta tecnología. Desde el año 2013 en que los astilleros españoles entregaron el último construido en Europa, la construcción de estos buques, hoy ya de 170.000 metros cúbicos de capacidad, solo se lleva a cabo en los tres países asiáticos líderes. Se da además la circunstancia de que estos buques son los únicos que pueden ofrecer alternativa al transporte de gas natural mediante gasoducto y proporcionan una libertad de elección de aprovisionamiento que no tiene el uso de gasoductos, especialmente en momentos de conflictos de cualquier tipo. Estos añaden además, como ya se ha dicho, el valor estratégico de transportar el producto energético menos contaminante en el medio plazo, no solo para la navegación sino para las necesidades energéticas terrestres.

La estrategia seguida por la UE, especialmente en las décadas de los años ochenta y noventa ha derivado en una calamidad industrial en el sector de la construcción naval europea: la mayoría de los grandes astilleros de buques mercantes cerrados, cientos de miles de empleos perdidos, enormes recursos disipados en ayudas de Estado a la reducción de capacidad, retiros anticipados y consiguiente pérdida de masa crítica y de conocimientos.
Mientras los astilleros de Japón y de Corea del Sur «compraban» cuota de mercado a precios con los que la industria europea no podía competir, las decisiones de la Comisión Europea ratificadas por el Consejo pivotaban alrededor de la concesión de ayudas de Estado directas a los contratos de construcción sin otra contrapartida que la ejecución de los mismos, y de una cuantía que, aunque limitada en cuanto al porcentaje, se suponía que enjugaba la diferencia entre los costes en el Extremo Oriente y los incurridos en la Unión Europea. La idea era que el paso en esos países asiáticos de construir en modo «mano de obra intensiva» a "capital y tecnología intensiva» equilibraría los costes con Europa y se podría competir sin ayudas. La realidad es que esto no ha sucedido así y que Japón, Corea del Sur y posteriormente China se hicieron con prácticamente todo el mercado y convirtieron a la industria de construcción naval europea en casi testimonial, con la excepción del segmento de los buques de crucero. Cuando los salarios se fueron aproximando, la potencia constructora en los países mencionados era tal que la situación nunca se revirtió.

De hecho, en el caso específico de Corea del Sur, el salario medio de un trabajador del sector era ya en 2009 de 46.000.000 de KRW, al cambio actual, 39.000 USD anuales, por encima de los que correspondían a la industria del automóvil, la aeronáutica, los semiconductores y la informática, en los que ya Corea era altamente competitiva. En el caso de Japón, el salario medio en 2016 era de 47.000 USD, y en el caso de China, 25.000 USD. Todos ellos promedios aproximados ${ }^{4}$.

Los países asiáticos mencionados y especialmente Japón y Corea han seguido comportamientos relativamente parecidos: han hecho concentraciones empresariales patrocinadas por sus Gobiernos, nacionalizando grupos en crisis (Corea) y modernizando o construyendo nuevas instalaciones más adecuadas a la evolución esperada del mercado; no han reducido capacidad

\footnotetext{
4 OECD (2016). OECD WP6 Korean Shipbuilding. https://www. glassdoor.com/Salaries/index.htm; CareerCross: Asia's Leading Recruitment. Revista Ingeniería Naval, 10/12, 1 y 2/2014. Accesible en https://dialnet.unirioja.es/servlet/revista?codigo $=5151$
} 
física en los grupos soportados directa o indirectamente por las políticas industriales de sus Gobiernos; no han abandonado mercados, sino que se han introducido en nuevos nichos o caladeros; han utilizado o creado entidades colaborativas en la industria marítima, tales como el Korea Institute of Ocean Science \& Technology (620 empleados), el Korea Marine Equipment Research Institute (150 empleados), o el Research Institute of Medium \& Small Shipbuilders, privadas o públicas, que han canalizado apoyos para mejorar la tecnología y la investigación y han ido mejorando su productividad, que hoy es excelente. El estatal Japan Ship Centre en Londres es otro ejemplo de promoción comercial y tecnológica orientada a la exportación de la industria naval japonesa puesto por el Estado al servicio de la industria del país. El caso de China es distinto por tratarse de un país con economía totalmente dirigida en el que tanto los grandes grupos constructores como navieros son de titularidad pública.

Otro de los grandes problemas de la industria de construcción naval europea es el de las herramientas existentes para regular la competencia internacional. El buque es un producto muy singular, tanto por la naturaleza de su mercado como por sus particularidades jurídicas, y esto crea enormes dificultades para que los problemas que surjan entre los países constructores se puedan dirimir de forma práctica en los foros comerciales internacionales, tales como la World Trade Organization (OMC). El único intento de llegar a un acuerdo sobre condiciones normales de competencia en la industria de construcción naval se desarrolló en la OCDE a finales del siglo pasado, con negociaciones que duraron años y que se materializaron en un acuerdo que jamás entró en vigor al no conseguirse finalmente su ratificación por parte de todos los países firmantes.

Pese a lo que hemos venido describiendo a lo largo de esta parte final del artículo, en el futuro la industria europea de construcción naval podría salir de la situación desoladora que muestran las cifras expuestas. Nos referimos al conjunto de la industria, es decir, a los astilleros y a todo el conglomerado técnico e industrial que diseña y manufactura los equipos y servicios de los buques o de los artefactos a construir.

Gran parte de esta producción a la que nos referimos sale de Europa con destino a los astilleros del Extremo Oriente que, debido a las cifras de negocio que ostentan, son sus principales clientes. Sin embargo, la realidad es que la tecnología de la industria marítima, entendida en todo su conjunto, hace de Europa, y especialmente de la UE, una región muy importante en el mundo en términos del valor añadido a la producción de buques y sistemas marinos de todo tipo, se construyan estos en Europa o fuera de ella ${ }^{5}$.

El mencionado estudio calcula su valor en 112.500 millones de euros, que representa un 23,3 por 100 del valor mundial (promedio anual 2010-2014). Sin embargo, la mayor parte de estos componentes va dirigida a nuestros competidores asiáticos ya que, por ser los más importantes constructores del mundo, son también los mayores demandantes de estos productos. El peligro de deslocalización tecnológica y de conocimientos hacia la competencia asiática es por tanto muy elevado, de tal modo que «toda» la industria puede acabar pasando a manos de nuestros competidores y más aún si estos siguen practicando políticas específicamente dirigidas a la industria marítima y al crecimiento de su valor añadido nacional.

El imparable tren del avance tecnológico debería ser utilizado por la industria europea de manera colaborativa y sin fisuras, lo cual la dotaría de una dimensión que pudiera permitirle competir.

La protección del medio ambiente y las cada vez más estrictas regulaciones, la revolución hacia energías más limpias, la creciente aplicación de la inteligencia artificial, de tecnologías de última generación, como fabricación por impresión 3D, los nuevos materiales, el uso de las tecnologías tipo block-chain y otras,

\footnotetext{
5 Sobre este tema se puede ver el Estudio de las nuevas tendencias de la globalización en la construcción naval y en la industria de sus componentes. Consecuencias para la política industrial europea, realizado por Balance Technology Consulting para la Comisión Europea.
} 
desarrolladas por una sociedad como la europea, en principio más comprometida con un desarrollo económico y social combinados con la calidad de vida y el bienestar, deberían actuar como palancas para recuperar una industria estratégica.

Hay que plantear qué es lo que se entiende en la Unión Europea por sostenibilidad económica y social a largo plazo, así como el aseguramiento de su soberanía industrial y de su aprovisionamiento estratégico, para que no se dé la circunstancia de que amanezca un día en el que nos demos cuenta de que todo lo que necesitamos se transporta por buques que han sido construidos, operados, poseídos y tripulados por nuestros máximos competidores comerciales. Desconocemos si entra dentro de la estrategia a largo plazo de la UE estudiar qué y cómo se tendría que actuar en caso de una situación de conflicto generalizado en la que los buques, responsables de la inmensa mayoría del transporte de aprovisionamientos vitales, no se pudieran construir en la menguada industria de construcción naval europea.

Aunque una situación como esa no parece vislumbrarse en la actualidad, la historia enseña que tal posibilidad siempre existe, y que la mejor manera de evitarla es estar preparado para ella.

\section{Conclusiones}

La industria europea de construcción naval ha ido perdiendo posiciones desde la década de los años setenta del pasado siglo. En la actualidad parece relegada a una existencia casi testimonial en lo que se refiere a la construcción de los buques responsables del transporte de la inmensa mayoría de las mercancías del comercio mundial. Parece que el valor estratégico de la industria ha sido mejor comprendido por los competidores, con los que siempre se ha desarrollado una batalla desigual en relación a lo que cada uno entendía como economía de mercado en este sector específico, en el que unos han establecido políticas nacionales al margen del mercado, y otros no.
Todavía parte de la soberanía tecnológica en este sector reside en Europa. Los nuevos desarrollos tecnológicos en la nueva era digital deberían ayudar a tomar las medidas para revertir o al menos equilibrar una situación que no se corresponde con la posición de la Unión Europea en el mundo ni con su capacidad estratégica de supervivencia a largo plazo. Todo dependerá de los recursos económicos, políticos y de colaboración que se destinen a este fin.

\section{Referencias bibliográficas}

[1] ATHENIAN SHIPBROKERS REPORTS (2017). Consultado el 5 de octubre de 2017 en www.atheniansa.gr

[2] AWES (2017). Annual Reports. Association of European Shipbuilders. Consultable en http://www.marinetalk.com/articlesmarine-companies/com/AWES-Association-of-European-Shipbuilders-AWE002.html

[3] BRUCE, G.J. y GARRARD, I. (1999). The Business of Shipbuilding. Lloyds of London Press.

[4] CLARKSON RESEARCH (2017). World Shipyard Monitor. Consultado el 1 de octubre de 2017.

[5] CLARKSON RESEARCH (2017). Shipping Intelligence Weekly. Consultado el 1 de octubre de 2017.

[6] COMMUNITY OF EUROPEAN SHIPYARDS ASSOCIATIONS (2017). Annual Reports. (CESA).

[7] INSTITUTO MARÍTIMO ESPAÑOL (2009). Economía del sector marítimo. Edición de IME-FEIN, 2009. https://www. ime.es/publicaciones/libro-1/

[8] KAUPPILA, J. (2015). Multi-year Expert Meeting on Transport, Trade Logistics and Trade Facilitation Sustainable Freight Transport Systems: Opportunities for Developing Countries. 14-16 October 2015. International Trade and Freight by 2050. International Transport Forum. Consultado en febrero de 2018 en http:// unctad.org/meetings/es/Presentation/Jari\%20KAUPPILA.pdf

[9] OCDE (1994). Agreement Respecting Normal Competitive Conditions in the Commercial Shipbuilding and Repair Industry. 21-12-1994.

[10] OCDE (2017). Analysis of Selected Measures Promoting the Construction and Operation of Greener Ships. Consultado en enero 2018 en $h$ ttp://www.oecd.org/sti/shipbuilding

[11] REVISTA INGENIERÍA NAVAL (2016). Asociación de Ingenieros Navales y Oceánicos de España. Consultados los números de 2016 y 2017.

[12] STOPFORD, M. (2008). Maritime Economics. $3^{\text {rd }}$ Ed. Routledge. London. New York.

[13] WIJNOLST, N. y WERGELAND, T. (2008). Shipping Innovation. Delft University Press. Rotterdam. 